\title{
SÉANCE DU 10 MARS 1922
}

PRÉSIDENCE DE M. P. GUÉRIN.

Lecture est donnée du procès-verbal de la dernière séance, dont la rédaction est adoptée.

Par suite de la présentation faite à cette séance, est proclamé membre de la Société :

M. Hinglais (Hermann), interne en pharmacie, Hospice des Incurables, avenue de la République, 7, à Ivry (Seine), présenté par MM. Guérin et Mascré.

M. le Président annonce ensuite trois nouvelles présentations. Il donne lecture d'une lettre de M. l'abbé Coste, remerciant la Société de son élection à la vice-présidence.

Il est présenté, au nom de Madame Philippe de Vilmorin, des rameaux fleuris de 40 arbustes à floraison hàtive, provenant de l'Arboretum de Verrières-le-Buisson :

Andromeda japonica Thunb. - Japon.

A. calyculata L. - Asie et Amérique boréales.

Armeniaca Mume De Vriese. - Japon.

A. Mume De Vriese var. flore pleno. - Japon.

Clematis Armandi Franch. - Chine.

Cornus Mas L. - Europe, Asie Mineure.

Corylopsis spicata Sieb. et Zucc. - Japon.

Daphne Mezereum L. - Europe, Asie boréaIe.

D. Mezereum L. var. album.

D. Laureola L. - Europe, Afrique boréale.

Erica carnea L. - Europe australe.

E. carnea L. var. alba.

Forsythia Fortunei Lindl. - Chine.

F. vitellina Hort.

F. suspensa Wahl - Japon.

F. viridissima Lindl. - Chine.

Garrya elliptica Dougl. - Californie.

T. LXIX.

(SÉANCES) 11 
Hamamelis arborea Ottolander. - Japon.

H. Zuccariniana Ottolander. - Japon.

H. rubra Hort.

Jasminum nudiflorum Lindl. - Chine.

J. Sieboldianum Blume. - Chine.

Lonicera Altmanni Regel et Schmal. - Turkestan.

L. fragrantissima Lindl. et Past. - Chine.

L. Standishii Carr. - Chine.

Nuttalia cerasiformis Torr. et Gray. - Amérique boréale occidentale.

Parrotia persica C. A. Mey. - Caucase.

Prunus cerasifera Ehrh. var. Pissardi Carr. - Perse.

P. triloba Lindl. - Chine.

P. (Amygdalus) dehiscens Kœhne. - Chine.

Rhododendron dauricum L. - Sibérie.

R. Davidii Franch. - Chine.

R. moupinense Franch. - Chine.

R. oreodoxa Franch. - Chine.

R. præco $x$ Hort. (ciliatum $\times$ dauricum).

R. sutchuenense Franch. - Chine.

Ribes fasciculatum Sieb. et Zucc. var. chinense, - Chine.

Shepherdia argentea Nutt. - Amérique boréale.

Stachyurus præcox Sieb. et Zucc. - Japon.

Sycopsis sinensis Oliver. - Chine.

\section{A noter particulièrement :}

Le Clematis Armandi, très belle espèce grimpante à fleurs blanches et beau feuillage persistant.

L'Hamamelis arborea, une des plus intéressantes espèces du genre par sa riche floraison.

Le Parrotia persica, très abondamment fleuri cette année.

L'Amygdalus dehiscens, grandes fleurs rose vif, espèce nouvelle d'introduction Wilson.

Les Rhododendron Davidii, fleur rose vif; R. moupinense, fleur blanche, espèce donnée comme épiphyte dans son pays natal; $R$. sutchuenense, belles et grandes fleurs blanc rosé.

Le Sycopsis sinensis, intéressant par son feuillage persistant, etc., etc.

On constatera que l'année en cours est loin de présenter la précocité extraordinaire de l'an passé. Beaucoup de ces espèces furent, en effet, présentées en fleur l'année dernière à la séance de la Société Nationale d'Horticulture de France du 27 janvier.

M. le Président, au nom de la Société, adresse ses plus vifs remerciements à Madame P. de Vilmorin.

L'ordre du jour appelle ensuite-communication des notes suivantes : 


\section{$2 \mathrm{BHL}$ Biodiversity Heritage Library}

1922. "Séance Du 10 Mars 1922." Bulletin de la Société botanique de France 69, 161-162. https://doi.org/10.1080/00378941.1922.10833416.

View This Item Online: $\underline{\text { https://www.biodiversitylibrary.org/item/93160 }}$

DOI: https://doi.org/10.1080/00378941.1922.10833416

Permalink: https://www.biodiversitylibrary.org/partpdf/161254

\section{Holding Institution}

Missouri Botanical Garden, Peter H. Raven Library

\section{Sponsored by}

Missouri Botanical Garden

\section{Copyright \& Reuse}

Copyright Status: Public domain. The BHL considers that this work is no longer under copyright protection.

This document was created from content at the Biodiversity Heritage Library, the world's largest open access digital library for biodiversity literature and archives. Visit BHL at https://www.biodiversitylibrary.org. 